\author{
J.D. Dorren \\ Biruni Observatory, Shiraz University, Shiraz, Iran. \\ E.F. Guinan \\ Villanova University, Villanova, Pennsylvania, 19085, USA
}

\begin{abstract}
Intermediate and narrow-band photoelectric observations obtained at Biruni and Villanova Observatories from 1977 to the present, when combined with previous data, strongly suggest that light increases of about $0.5 \mathrm{mag}$ occur at 6 year intervals. Possible explanations are considered.
\end{abstract}

I.

\title{
INTRODUCTION
}

The X-ray pulsator X Persei $(4 U$ 0352+30) is an intriguing object at both X-ray and optical wavelengths. From the X-ray data a period of 13.9 minutes now appears well-established, but early suggestions of a 22.4 hour periodicity remain unconfirmed (White et al.,1976, Becker et al., 1979). The nature of the X-ray emission is unusual; the source is about 3 orders of magnitude weaker than other X-ray binary pulsars, and the spectrum contains a strong high-energy X-ray component (Mushotzky et al., 1977). Long known as an irregular variable, $X$ Per has been classified as a Be star with an 09.5 (III-IV)e spectral type and showing broad hydrogen emission and absorption features (Brucato and Kristian 1972, Hutchings et a1., 1975). A 581-day period is indicated by the radial velocity study of Hutchings et a1., (1975). This has been variously interpreted as an orbital period (about a black hole) or an apsidal period, the models having in common accretion on to a neutron star as the source of the pulsed X-rays. In 1977 an increase in brightness of the star was observed by Kalv (1977) and Guinan et al., (1978), and by April 1979 we had accumulated sufficient evidence to suggest that $X$ Per undergoes periodic ( 6-year) outbursts (Dorren et al., 1979a).

2 .

\section{PRESENT OBSERVATIONS}

Photoelectric observations of X Per commenced at Biruni Observatory in August, 1977 using intermediate and narrow-band $\mathrm{H} \alpha$ filters and a Stromgren $u$ filter $(\lambda 3500)$ and continued up to the present (September 1979) both there and at Villanova University (using an $\mathrm{H} \alpha$ filter set matching that at Biruni and a pair of intermediate and narrow-band filters centered on the OI $\lambda 7774$ feature). Descriptions of the filters and 361

M. J. Plavec, D. M. Popper and R. K. Ulrich (eds.), Close Binary Stars: Observations and Interpretation, 361-365. Copyright $\odot 1980$ by the IAU. 
instrumentation at Biruni and Villanova are to be found in Guinan et al., (1979) and Guinan and McCook, (1974) respectively. A detailed discussion of this very extensive material (a total of 110 nights in $\mathrm{H} \alpha$ ) will be published elsewhere (Dorren et al., 1979b).

To trace the history of $X$ Per we extract from the compilation of Mook et al., (1974) the photoelectric V and B-V observations from 1964 to 1973. A comparison of our data with the $V$ band may be made by determining the wavelength dependence of the light variation from the 3 bandpasses $\lambda 6685, \lambda 7790$ and $\lambda 3500$ and then scaling the $\lambda 6585$ data to the effective $V$ wavelength $(\lambda 5500)$. The $V$ observations from 1964 to the present, Kalv's data, and the interpolated Biruni-Villanova $V$ measures are displayed in the figure, together with the B-V index where observed. Also included are $\mathrm{V}$ and $\mathrm{B}-\mathrm{V}$ observations obtained during January 1975 by Margon et a1. (1976). To avoid overcrowding, only a portion (about one third) of the Biruni-Villanova data was plotted. From the data obtained from 1974 onward, it is clear that there was a period of relatively constant light, perhaps slowly decreasing up to the spring of 1977 when $\mathrm{V}=+6.71 \mathrm{mag}$. Thereafter there was a fairly sharp increase in light by August 1977, indicated both by Kalv's data and our own. Maximum brightness of around $V=+6.38$ mag occurred in February 1978 and was followed by a decline of similar slope with a pause about 120 days long in mid-1978. The decline continued to a brightness of $V=+6.79 \mathrm{mag}$, the faintest the star has been since 1903, and the most recent data (September 1979) indicates that the decline has slowed or possibly halted. This may be the start of another interval of roughly constant light similar to that which occurred between 1973 and early 1977. An inspection of the figure indicates two other maxima occurring in mid-1966 and early 1972 which appear to be brighter than the most recent one, and the three occur at intervals of approximately 6 years. Although there is no data from 1968 to 1972 , the whole figure is suggestive of a repetitive pattern of outbursts lasting roughly 2 years separated by intervals of nearly constant light of about 4 years duration. It is interesting to note that, at times of constant low light, the $B-V$ index is bluest at $B-V \approx+0.16$ while near the maxima, $B-V=+0.25$ to +0.30 . Additional UBV data has been published by Harris (1956) where $\mathrm{V}=6.13, \mathrm{~B}-\mathrm{V}=+0.29$ and by Hiltner (1956) where $\mathrm{V}=6.07, \mathrm{~B}-\mathrm{V}=+0.31$. The dates of observation, though uncertain, are probably 1954 or 1955 (Harris) and 1954 (Hiltner). These colors and magnitudes suggest that the star is near maximum light, which is consistent with the 6-year cycle indicated by the later data. The only other reliable material is the visual photometry of Müller and Kempf (1907) from 1888-1902 which shows cyclic behavior in which the maxima occur near times expected with the 6-year period, in 1894 and 1900 .

3 .

\section{DISCUSSION}

The evidence for a 6-year periodic behavior is sufficiently compelling to warrant a discussion of possible explanations of the phenomenon. The two most likely mechanisms would appear to be (a) the ejection of an envelope by the 09 star and (b) accretion on to a compact object (probably a neutron star) leading to nuclear burning. The first 


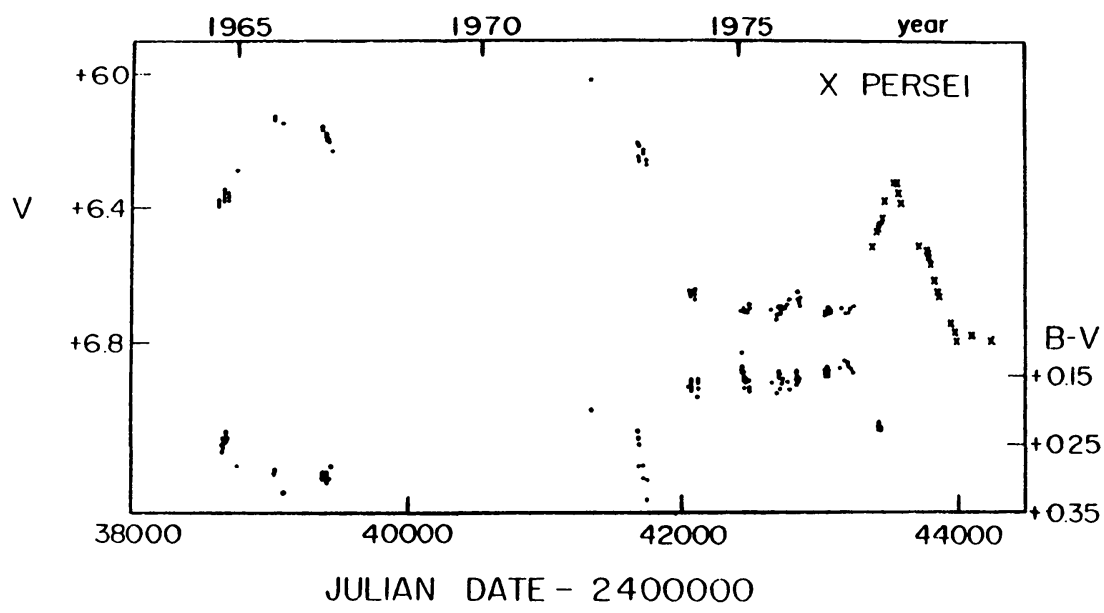

Fig. 1. V-band photometry of X Persei. The crosses are the interpolated Biruni-Villanova data points.

possibility is supported by the fact that X Per has long been considered to be a Be star with broad hydrogen emission and absorption features. The increase in light would then be associated with the ejection of an envelope, initially optically thick, but which, as it expands outwards, becomes less dense and less luminous. However no spectroscopic evidence for velocity changes corresponding to shell ejection have been reported, and such periodic behavior or range of light fluctuation is not known to be characteristic of other Be stars (Underhi11, 1960).

There are similarities between the photometric behavior of $\mathrm{X}$ Per and that of recurrent novae and symbiotic stars. Periodic outbursts followed by longer intervals of quiescence on time scales of months to tens of years are characteristic of the recurrent novae and, to a lesser extent, of some symbiotic stars. Both of these types are believed to be binaries with orbital periods of hours for the former and typically from 100 to 1000 days for the latter. The outbursts are believed to be produced by accretion on to a more compact companion (a white dwarf) leading to repetitive thermonuclear shell flashes. The light changes in the novae are typically $5 \mathrm{mag}$ or more, in symbiotic stars 2-3 mag, while for $X$ Per we observed a change of about $0.5 \mathrm{mag}$. Hence a possibility which should be investigated further is a model for X Per in which there is an 09 star with a neutron star companion where the light changes arise from the burning of material accreted on to the neutron star. Changes in brightness of the neutron star of several orders of magnitude would result in a relatively small overall change in light due to the presence of the highly luminous 0-star which dominates the total light of the system, a circumstance which does not occur in the novae. This model 
is in accord with others in which $13.9 \mathrm{~min}$. X-ray period is associated with the rotation of a neutron star, and the spin-up rate and low X-ray luminosity point to a relatively small accretion rate (Becker et al., 1978 and refs. therein). There is no explanation for a 22.4 hour modulation in this model but evidence for this period is rather weak. Continuing observations at all wavelengths are clearly desirable. An examination of the existing $\mathrm{X}$-ray and spectroscopic data for flux changes over a 6-year period would be particularly interesting.

\section{REFERENCES}

Becker, R.H., Boldt, E.A., Holt, S.S., Pravdo, S.H., Robinson-Saba, J., Serlemitsos, P.J., and Swank, J.H.: 1979, Astrophys. J. Letters 227, pp 21-24.

Brucato, R.J., and Kristian, J.: 1972, Astrophys. J. Letters 173, pp. 105-107.

Dorren, J.D., Guinan, E.F., and McCook, G.P.: 1979a, I.A.U.Circ. No. 3352

Dorren, J.D., Guinan, E.F., McCook, G.P. and Siah, M.J.: 1979b, in preparation.

Guinan, E.F., Dorren, J.D., Siah, M.J. and Koch, R.H.: 1979, Astrophys. J. 229, pp. 296-303.

Guinan, E.F. and McCook, G.P.: 1974, Pub. Astron. Soc. Pac. 86, pp. 947951.

Guinan, E.F., McCook, G.P. and Dorren, J.D.: 1978, I.A.U.Circ. No. 3182. Harris, D.L.: 1956, Astrophys. J. 123, pp. 371-372.

Hiltner, W.A., 1956, Astrophys. J. Supp1. 2, pp. 389-462.

Hutchings, J.B., Cowley, A.P., Crampton, D., Redman, R.0.: 1974 , Astrophys. J. Letters 191, pp. 101-104.

Hutchings, J.B., Crampton, D., and Redman, R.0.: 1975, Monthly Notices Roy. Astron. Soc. 170, pp. 313-324.

Kalv, P.: 1977, Inf. Bull. Var. Stars. No. 1359.

Margon, B., Bowyer, S., and Penegor, G.: 1976, Monthly Notices Roy. Astron. Soc. 176, pp. 217-223.

Mook, D.E., Boley, F.I., Foltz, C.B., and Westpfah1, D.: 1974, Pub. Astron. Soc. Pac. 86, pp. 894-898.

Müller, G., and Kempf, P.: 1907, Astron. Nachr. 175, pp. 162-166.

Mushotzky, R.F., Roberts, D.H., Baity,W.A., and Peterson, L.E.: 1977, Astrophys. J. 211, pp. 129-133.

Underhil1, A.: 1960, in Stellar Atmospheres, ed. J.L. Greenstein, Univ. of Chicago Press.

White, N.E., Mason, K.O., Sanford, P.W. and Murdin, P.: 1976, Monthly Notices Roy. Astron. Soc. 176, pp. 201-215.

\section{COMMENTS FOLLOWING DORREN AND GUINAN}

Henrichs: The stability of the 13.9 min. X-ray pulse period indicates a neutron star rather than a white dwarf (Mason 1977;

Rappaport and Joss 1977; Rappaport 1979). This is perhaps the most firm requirement for any model. 
Guinan: I agree with you that the X-ray source has characteristics of a neutron star rather than a magnetic white dwarf.

Kaitchuck: It is perhaps worth mentioning that the rapid $\mathrm{H} \alpha$ variations I reported on this morning all occurred during the last brightening of $X$ Per. The observations made after it faded have shown a constant line strength.

Chambliss: Is there a reliable radial velocity curve or an estimate of $f(\bar{M})$ for X Persei?

Guinan: Yes. A radial velocity study of $X$ Per has been published by Hutchings et al. in 1974 .

Mazeh: Can you suggest an observational test to verify your model against the other models?

Guinan: Yes. The 6-year light variation apparently found should have an X-ray counterpart. 